\title{
The Integrated Autonomous Underwater Navigation System based on Open Platform for Robotic Services
}

\author{
Youngho Choi* \\ Korea Institute of Robot and Convergence \\ Applied Technology Division \\ Pohang, Korea \\ *Corresponding author \\ Jinho Suh \\ Korea Institute of Robot and Convergence \\ Applied Technology Division \\ Pohang, Korea
}

\author{
Jungwoo Lee \\ Korea Institute of Robot and Convergence \\ Applied Technology Division \\ Pohang, Korea \\ Jongdeuk Lee \\ Korea Institute of Robot and Convergence \\ Applied Technology Division \\ Pohang, Korea
}

\begin{abstract}
This paper introduces how to easily integrate the modular navigation system for the autonomous underwater vehicle (AUV), which consists of the various hardware and software modules. This paper is focused on how to build the integrated underwater navigation module which is applicable to various types of underwater vehicle rather than the core algorithm for the autonomous underwater navigation. The autonomous underwater navigation system consists of many software, hardware components and the systematic integration of these components is very important to build up the efficient navigation system within the given resources. In order to achieve this goal, we introduce the systematic integration manner developing the modular autonomous underwater navigation system based on Open Platform for Robotic Service (OPRoS) [1].
\end{abstract}

Keywords-autonomous; underwater; navigation; modular; OPRoS.

\section{INTRODUCTION}

Autonomous Underwater Vehicles (AUVs) are useful tools for commercial and military tasks underwater, under-ice or in other environments [5]-[7] and have portable energy and selfcontrol ability which make them different from remote operate vehicles (ROVs). As the capabilities of these platforms continue to expand and they continue to mature as operational assets, navigation remains a fundamental technological component.

In the last few decades, many companies and institutes have built about 200 AUVs, such as the well-known REMUS by WHOI [8] or MIT Sea Grant's Odyssey [9].

As the applications of AUVs are spreading to deeper seas and longer distances, high accuracy navigation capability will play a vital role. Navigating an AUV presents unique challenges to the researcher and the practitioner.

AUVs consist of many sciences and technologies, among which navigation is one of the most important techniques. On the other side navigation is the precondition of long distance and autonomous voyage for AUV, which ensure that AUV can complete various tasks, such as investigating resource, laying pipeline, exploring some place and so on. The tactics missions of AUV and voyage surroundings require AUV possess not only precision but also capability toward the environment disturbance.

AUVs generally use inertial navigation systems (INS). Unlike the navigation system relying on GPS on the ground, underwater navigation system cannot use this GPS because electromagnetic signals decay very quickly in the water. Therefore underwater navigation is more difficult than that of on the ground. In addition, it is very difficult to obtain prior maps of large-scale complex underwater environments. For all the reasons mentioned above, autonomous underwater navigation is considered one of the most challenging issues for AUVs.

In this paper, we focused on how to build the integrated underwater navigation module which is applicable to various types of underwater vehicles rather than the navigation algorithm, itself.

The rest of this paper is organized as follows. Section 2 introduces the hardware and software architecture of the proposed autonomous underwater navigation system. In section 3, the experimental result acquired from the implemented prototype is described and then section 4 concludes the paper.

\section{OVERALL DESIGN OF THE NAVIGATION SYSTEM}

The proposed autonomous underwater navigation system is toward the modularized system which is applicable to various types of underwater vehicles. It basically consists of the dual CPU hardware system conducting various algorithm loops and software framework for integrating various software modules. The details of hardware and software architecture are described in the following subsections.

\section{A. Hardware Architecture}

Navigation system consists of various software modules such as path planner, localizer, task manager and so on. In order to make these software modules work stably, the 
distribution of computing resource is very important because the computing power of CPU is limited. Therefore, the proposed system consists of dual industrial single board computer (SBC) and we distributed software modules to two SBCs to the end that each SBC uses the similar computing power while conducting its own given work. In addition to this, we add the embedded board which is for managing power and interfacing with various types of sensors and network modules. Overall hardware architecture is shown in Figure 1. Ethernet switching hub is installed in this embedded board for networking between two SBCs and external devices. Figure 2. Shows the 3D design of the frame for integrating 3 common hardware modules and sensors for the underwater navigation such as IMU, depth sensor, etc. This frame can be easily applied to circular shaped pressure vessel of underwater navigation platform. Figure 3 shows the SBC specification and interface board which is interfacing between SBC connectors and the backbone socket of the case.

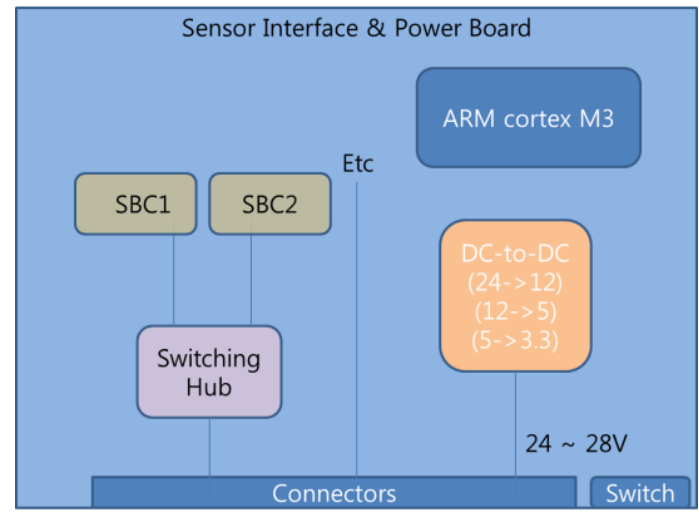

FIGURE I. OVERALL HARDWARE ARCHITECTURE
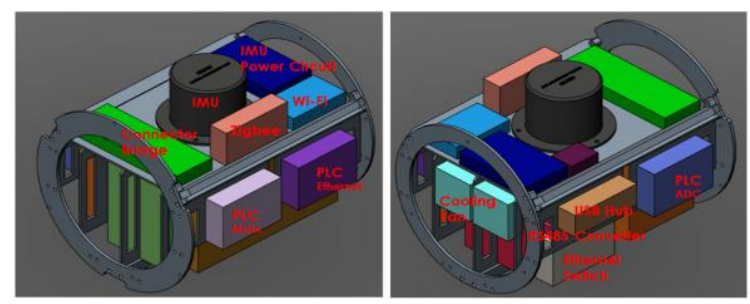

FIGURE II. FRAME DESIGN FOR THE HARDWARE INTEGRATION

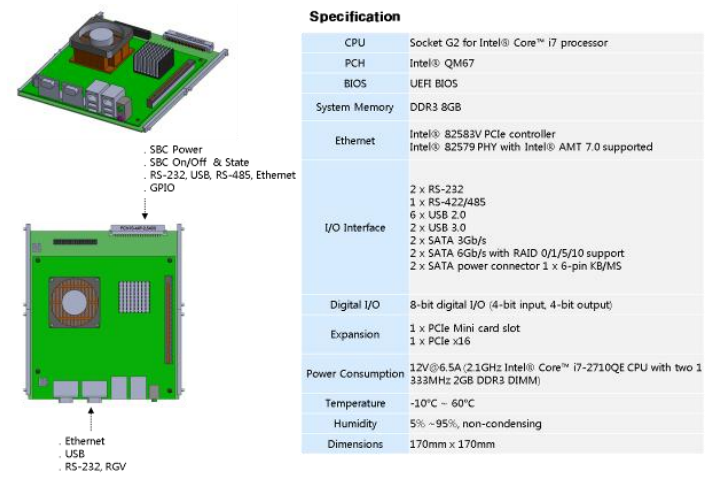

FIGURE III. SBC SPECIFICATION AND INTERFACE BOARD DESIGN LAYOUT
In order to interface with various types of sensors, SBC has many type of I/O interface and in order to simplify power system, we choose $12 \mathrm{~V}$ single voltage working model. Figure 4 shows the layout and specification of the embedded board. It consists of the power managing unit, interfacing unit and networking unit. The power managing unit is for making various voltage levels by using DC to DC converting chips. The interfacing unit consists of various types of sockets which is connected to various types of the external sensors and devices. The networking unit consists of Ethernet switching hub and communication bus. Through this bus, Each SBC is able to communicate with each other and external devices with the Ethernet cable.

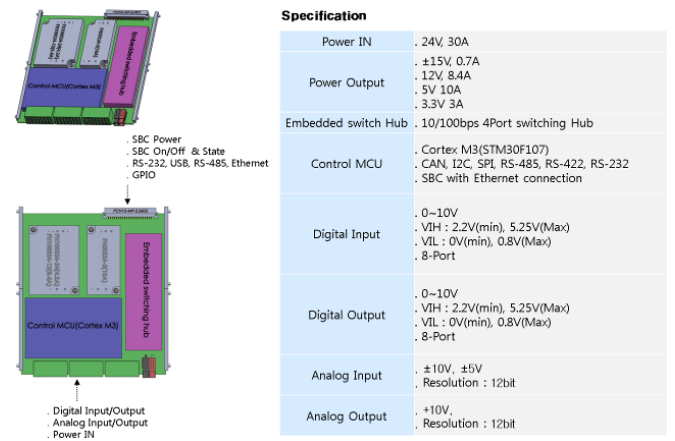

FIGURE IV. EMBEDDED BOARD SPECIFICATION AND DESIGN LAYOUT

\section{B. Software Architecture}

As depicted above, the autonomous underwater navigation system consists of many software components and these components have to be systematically interlocked with each other component in order to conduct the goal of autonomous navigation. The software architecture of the proposed navigation system is basically 4 layered structure as shown in Figure 5.

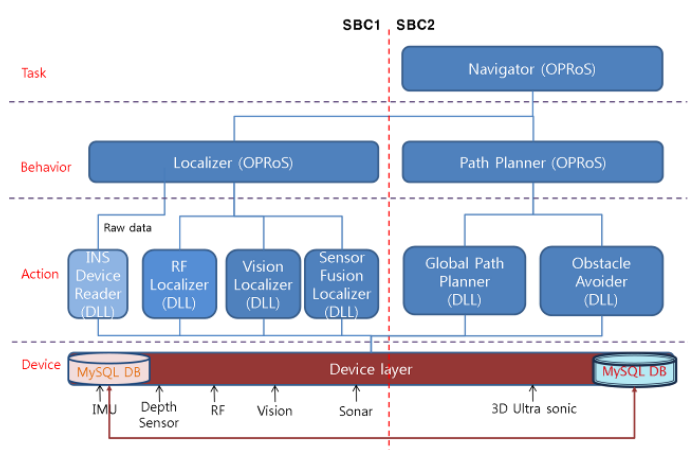

FIGURE V. OVERALL SOFTWARE ARCHITECTURE

The device layer is for abstracting the connection between the physical sensor device and software components using its data. i.e., the software components do not have physical connection with sensors but the device layer is connected with physical sensors and the software component acquires the sensor data from the device layer. By using this structure, the software components can share the sensor data from the same physical sensor.

To this end, we adopted memcached server to share the big 
size data such as 3D sonar data, high resolution image data, etc. Figure 6 shows the architecture for sharing the big data through the memcached server.

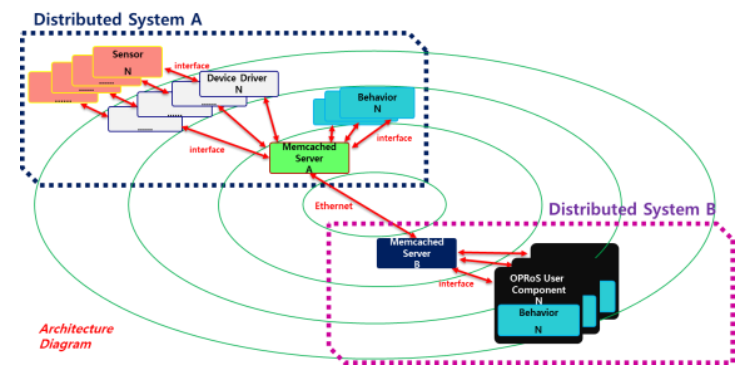

FIGURE VI. ARCHITECTURE OF THE MEMCACHED SEVER FOR SHARING THE BIG DATA BETWEEN DISTRIBUTED SYSTEMS

The action layer is for the unit algorithm modules which is the element of the functional software module of the behavior layer. The software components in behavior layer are implemented by composing the dll-type compiled library of the action layer. The task layer is the top most software component of the proposed navigation system and it is composed with the services from the software component of the behavior layer. The components of the behavior and the task layer require the intercommunication between other components which are working on the other machines. Therefore, we adopted Open platform for Robotic Service (OPRoS) as the software framework for the software integration. These components are implemented according to the standard of OPRoS component and communicate with each other through data, service and event channel as shown in Figure 7 and their data flow between these components is depicted in Figure 8.

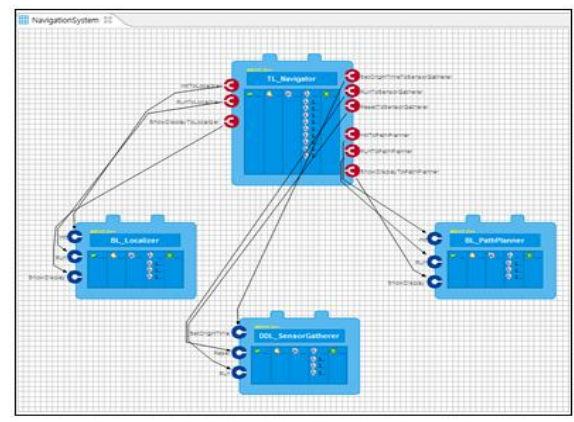

FIGURE VII. SERVICE CONNECTION BETWEEN OPROS COMPONENTS

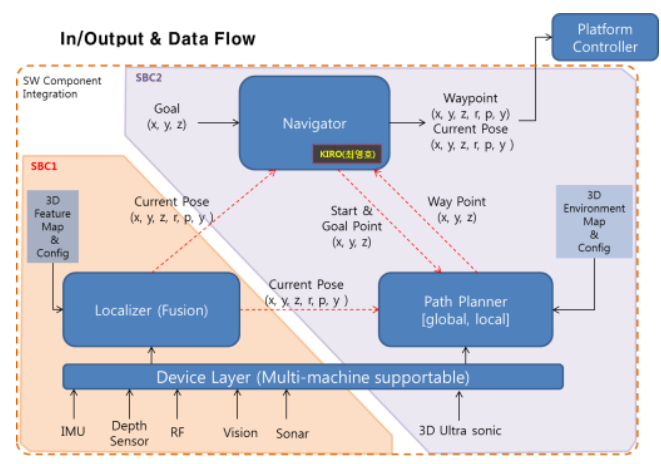

FIGURE. VIII. DATA FLOW BETWEEN OPROS COMPONENTS

\section{EXPERIMENTAL RESULTS}

\section{A. Prototype System}

We implemented the special prototype for testing the hardware and software stability and performance of the proposed modularized autonomous underwater navigation system and applied core algorithm such as localizer and path planner as shown in Figure 9. This prototype is specially designed for the application to PSUO-II which is AUV platform developed in the Korea Institute of Robot and Covergence (KIRO) as shown in Figure 10.

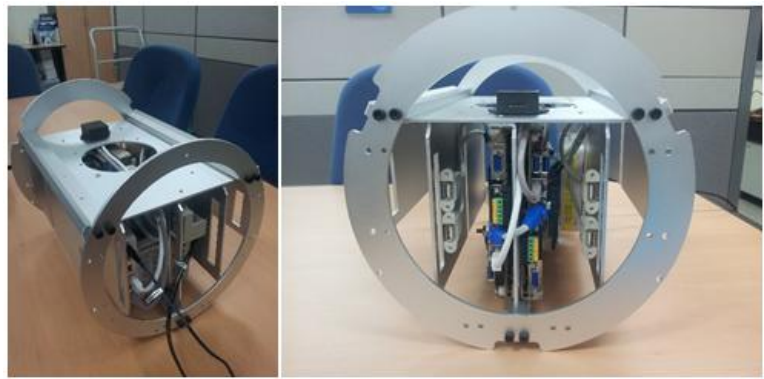

FIGURE IX. PROTOTYPE OF THE PROPOSED SYSTEM

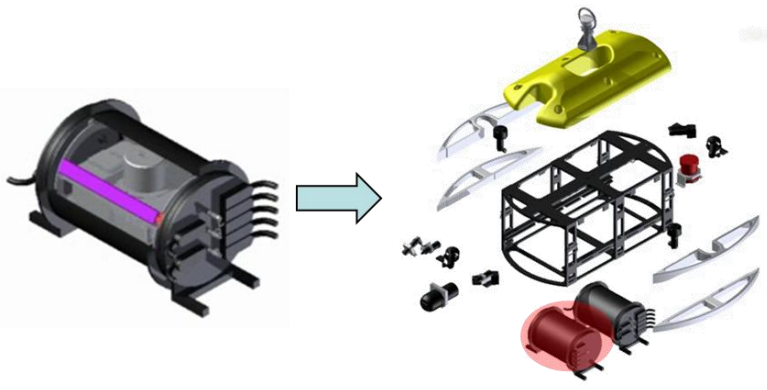

FIGURE X. APPLICATION OF PROTOTYPE TO PSURO-II

\section{B. Stability and Performance Test}

As mentioned above, we adopted two core navigation algorithm modules, localizer and path planer to our prototype. In addition to this, we added navigator component for the interface between user and these two algorithm modules.

Then, we test the error rate and time delay of data, service and event communication between these three components.

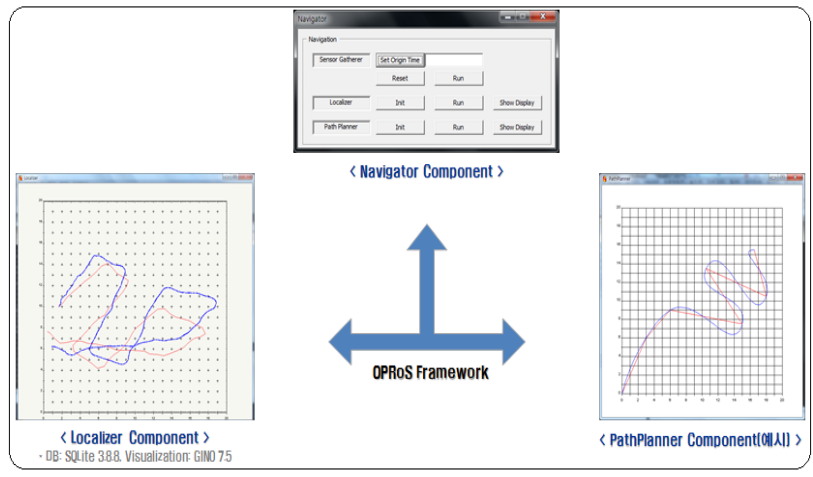

FIGURE XI. INTEGRATED COMPONENTS BASED ON OPROS

Figure 11 shows the implemented components and their integration using OPRoS. Table 1 shows the test result. This 
results is calculated after executing the same test scenario 100 times.

TABLE I. TEST RESULT USING THREE COMPONENTS.

\begin{tabular}{cc}
\hline Error rate [\%] & 0.65 \\
\hline Time delay [msec] & 5 \\
\hline
\end{tabular}

\section{CONCLUSION}

In this paper, we introduced the design method of navigation system for the autonomous underwater vehicle (AUV), which includes overall navigation architecture, hardware and software structure. This paper is focused on how to build the integrated underwater navigation module which is applicable to various types of underwater vehicle rather than the navigation algorithm, itself.

In order to test the concept of the proposed system, we implemented the prototype without the real working algorithms and tested it by using random data. This test result shows that our proposed system fulfils the required specification.

Future work is implementing real system with the real working algorithm and then applying it to the real AUV platform.

\section{ACKNOWLEDGMENT}

This work was supported by grant No. 10043928 from the Industrial Source Technology Development Programs of the MOTIE (Ministry Of Trade, Industry \& Energy), Korea.

\section{REFERENCES}

[1] http://ropros.org

[2] Y. Sun, L. Wan, Y. Pang, "Design of the Embedded Navigation System of Autonomous Underwater Vehicle based on the VxWorks ," ICCA, pp. 2919-2924, 2007.

[3] D. Brutzman, M. Burns, "NPS Phoenix AUV software integration and in-water testing," AUV, pp. 99-108, 1996.

[4] D. Barnett, S. MacClaran, E. Nelson, "Architecture of the Texas A\&M Autonomous Underwater Vehicle Controller," AUV, pp. 231-237, 1996.

[5] Yiping Li, Yesterday, "Today and tomorrow of underwater robotic," Automation panorama, vol. 9, pp. 56-59, 2002.

[6] Xisheng Feng, Yongkuan Liu, "Status of AUV's R \& D and trend," High technique message. vol. 9, pp. 55-59, 1999.

[7] Engfang Sang, Yongjie Pang, Hongyu Bian, "Technology of underwater

[8] Allen, B.; Stokey, R.; Austin, T.; Forrester, N.; Goldsborough, R.; Purcell, M.; von Alt, C. REMUS: A Small, Low Cost AUV; System Description, Field Trials and Performance Results. In Proceedings of the Oceans Conference, Halifax, NS, Canada, 6-9 October 1997; pp. 9941000.

[9] Bovio, E.; Cecchi, D.; Baralli, F. Autonomous underwater vehicles for scientific and naval operations. Ann. Rev. Control 2006, 30, 117-130. 